\title{
Latent tuberculosis infection as a target for tuberculosis control
}
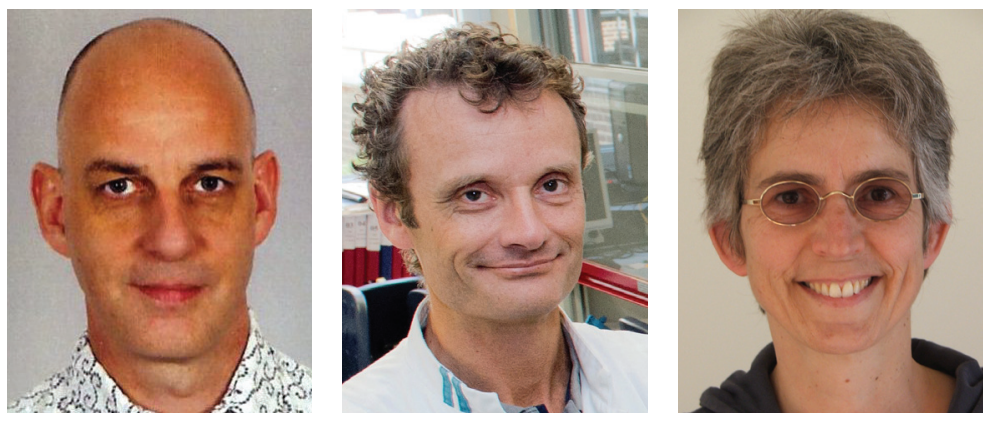

Frank van Leth ${ }^{*, 1}$, Reinout van Crevel $^{2} \&$ Miranda Brouwer ${ }^{3}$

WHO recently published guidelines for identifying and treating individuals with latent tuberculosis infection (LTBI) in high- and middle-income countries with a low incidence of active tuberculosis as part of the 'End TB Strategy' for the post-2015 era of tuberculosis control [1]. Systematic screening for LTBI is advised for immunocompromised individuals and contacts of patients with tuberculosis, in whom the risk of progression from latent infection to active diseases is increased, and for whom prevention of progression through adequate treatment has the highest impact. For migrants from countries with a highincidence of tuberculosis and healthcare workers, LTBI screening is encouraged.

Diagnosing LTBI, however, is hampered by the absence of an adequate reference test. The currently used tuberculin skin test (TST) and Interferon-gamma release assays (IGRAs) measure the immunological response to Mycobacterium tuberculosis-associated antigens in vivo and ex vivo, respectively. IGRAs were seen as a major improvement in diagnosing LTBI as they would, among other characteristics, suffer less from immunosuppression associated anergy than TST. Unfortunately, this turned out not to be completely true. In a multicenter European study, Sester et al. included 1537 patients with different aetiologies of immunosuppression who were tested for LTBI during routine care. Indeterminate test results were seen in up to $20 \%$ of the participants, depending on the level of immunosuppression [2]. This study confirmed findings from earlier studies in HIV-infected populations only $[3,4]$.

In immunocompromised persons there seems to be no clear association between TST/IGRA test result and risk of progression to tuberculosis. In the study by Sester et al., 11 individuals developed tuberculosis within 5 years of the initial test, of whom ten were HIV-infected and one was a solid organ transplant recipient. Six out of these 11 patients had either a negative or indeterminate LTBI test result, while only two had a positive test with both IGRA and TST [2]. Although reinfection could not be ruled out, four of these six patients developed tuberculosis within 2 years of the initial test, which is

'Department of Global Health, Academic Medical Center, Universty of Amsterdam, Amsterdam Institute for Global Health \& Development, Amsterdam, The Netherlands

2Department of Medicine, Radboud University Medical Center, Nijmegen, The Netherlands

${ }^{3}$ PHTB Consult, Tilburg, The Netherlands

*Author for correspondence: Tel.: +31 20566 7800; f.vanleth@aighd.org

\section{KEYWORDS}

- infection control • latent tuberculosis infection $\bullet$ screening

- tuberculosis

“...screening and treating latent tuberculosis infection in the immigrant population in low-endemic settings could have a substantial larger impact on reducing tuberculosis incidence in low-endemic countries." 


\section{“The attention given to latent tuberculosis infection screening in immunocompromised individuals and tuberculosis contacts seems to be at odds with the epidemiology in low-endemic countries..."}

generally considered as progression to disease rather than reinfection. There was no progression to tuberculosis in the populations with the highest prevalence of LTBI (rheumatoid arthritis and chronic renal failure). An earlier systematic review in HIV-infected patients showed a similar poor identification of patients at risk for progression [5].

The purpose to test for LTBI in close contacts of identified tuberculosis patients is to identify recent infection due to recent exposure. Early identification and subsequent provision of preventive therapy can significantly reduce the risk of progression to active disease. Information on progression to active disease in a population of tuberculosis contacts is scarce. Diel et al. reported disease progression in 19 of 198 IGRApositive contacts out of 954 close contacts tested. There was no progression in IGRA-negative contacts. By contrast, of the 242 TST-positive contacts, ten progressed to active disease, while nine contacts with disease progression were TST negative [6].

Providing preventive therapy to individuals with a positive test result on TST/IGRA has proven to be effective in reducing progression to tuberculosis regardless of the drug regimen taken [1], especially in countries with a low prevalence of tuberculosis where the risk of reinfection is minimal. However, the substantial number of immunocompromised patients or tuberculosis contacts with a positive LTBI test not progressing to disease would result in a considerable overtreatment. The combination of a high prevalence of positive tests, the overall low frequency of progression to active disease in lowendemic settings and the poor ability of IGRA and TST to identify those at highest risk for progression, makes that the effectiveness of blanket testing for LTBI and preventive therapy in these high-risk groups needs to be carefully evaluated, despite the potential impact on preventing new tuberculosis cases.

For HIV-infected individuals there are additional arguments against blanket LTBI testing and treatment in settings with a low prevalence of tuberculosis. These settings are mainly high-income countries where HIV is often diagnosed early at relatively high CD4 cell counts. Early start of combination antiretroviral therapy (cART) in these patients substantially decreases the risk of tuberculosis, with patients starting cART with high numbers of CD 4 cells $\left(>500 / \mathrm{mm}^{3}\right)$ having at least a tenfold lower risk of developing tuberculosis compared with those starting with $<50-100 / \mathrm{mm}^{3}$ CD 4 cells $[7,8]$. An additional factor is the changing epidemiology of HIV in high-income countries, where the relative contribution of injecting drug use to the spread of HIV is declining, while the proportion of men having sex with men (carrying a much lower tuberculosis risk) among newly diagnosed patients is increasing. As a result, less than $1.5 \%$ of HIV infected individuals receiving cART in settings with a low prevalence of tuberculosis develop active tuberculosis at any point in time [4,9-11]. The remaining risk of progression seems largely confined to TST-positive individuals originating from countries with high prevalence of tuberculosis, and is around four-times higher compared with those from low-endemic settings $[4,12]$.

The attention given to LTBI screening in immunocompromised individuals and tuberculosis contacts seems to be at odds with the epidemiology in low-endemic countries, where tuberculosis occurs disproportionately more often in the immigrant population as a result of reactivation of earlier acquired LTBI rather than recent transmission [13,14]. National screening programs for immigrants are therefore in place in many of these countries, albeit varying widely in content and setting. The emphasis of immigrant screening is to detect active tuberculosis, while systematic screening for LTBI is not performed routinely. A study in The Netherlands by Mulder et al., showed that testing for LTBI with either TST or IGRA as part of a mandatory immigrant screening, enabled the identification of those immigrants at high risk for progression to active disease. The risk of progression to tuberculosis was 15-times higher for immigrants with a positive versus a negative TST, while this was 18-times for immigrants with a positive versus a negative IGRA [15]. Immigrant screening based on identification of LTBI was cost effective as compared with the conventional strategy of identifying active disease in the UK [16] and The Netherlands [Mulder C et AL., Unpublished Data].

Concurrent with strategies to identify and treat individuals with LTBI, there is a need to reduce actual transmission and prevent infection from occurring. This is especially important in healthcare facilities, where nosocomial infection has been described in both low- and high-endemic settings. A meta-analysis for the incidence of LTBI showed an annual risk of infection in healthcare workers of $3.8 \%$ in 
low-endemic countries, and $7.2 \%$ in highendemic countries, with more than $1 \%$ of the healthcare workers in high-endemic settings developing active tuberculosis each year [17].

Measures to prevent nosocomial transmission include prompt identification of (presumptive) tuberculosis patients and separation of these patients from others, use of ventilation systems including natural ventilation and the use of particulate respirators to protect wearers from inhaling the tuberculosis bacilli. These type of measures to protect healthcare workers could potentially decrease the incidence of tuberculosis in healthcare workers by as much as $89 \%$ in high-endemic setting [17].

Studies have shown that implementation of such measures is quite challenging due to lack of necessary tools and unambiguous guidelines, and suboptimal motivation on the side of healthcare workers who need to implement these measures, among others [18,19]. A study in all 24 healthcare facilities for multidrugresistant tuberculosis in South Africa, showed that infection-control practices varied widely between facilities, and adequate knowledge of these practices was lacking in a large group of healthcare workers [18]. Focus group discussions in Mozambique identified that healthcare workers find it difficult to separate clinic attendants with cough from those without since they perceive it as discriminatory and contributing to stigmatization. At the same time, providing enough ventilation in the consultation room by opening doors was seen as interfering with the patient's privacy [20]. Monitoring of LTBI and incidence of tuberculosis in healthcare workers is the best method available to verify ongoing transmission in these settings.

Tuberculosis-control activities should be regularly evaluated for effectiveness against changing epidemiological information. Although identification of LTBI in immunocompromised patients and contacts of tuberculosis patients can be seen as good clinical practice; screening and treating LTBI in the immigrant population in lowendemic settings could have a substantial larger impact on reducing tuberculosis incidence in lowendemic countries. There is a strong need for providing a conducive environment for implementing infection control measures and monitoring their effectiveness in healthcare facilities.

\section{Financial \& competing interests disclosure}

The authors have no relevant affliations or financial involvement with any organization or entity with a financial interest in or financial conflict with the subject matter or materials discussed in the manuscript. This includes employment, consultancies, honoraria, stock ownership or options, expert testimony, grants or patents received or pending, or royalties.

No writing assistance was utilized in the production of this manuscript.

\section{References}

1 World Health Organization. Guidelines on the management of latent tuberculosis infection. http://apps.who.int

2 Sester M, van Leth F, Bruchfeld J et al. Risk assessment of tuberculosis in immunocompromised patients. A TBNET study. Am. J. Respir. Crit. Care Med. 190(10), 1168-1176 (2014).

3 Aichelburg MC, Rieger A, Breitenecker F et al. Detection and prediction of active tuberculosis disease by a whole-blood interferon-gamma release assay in HIV-1-infected individuals. Clin. Infect. Dis. 48(7), 954-962 (2009).

4 Elzi L, Schlegel M, Weber R et al. Reducing tuberculosis incidence by tuberculin skin testing, preventive treatment, and antiretroviral therapy in an area of low tuberculosis transmission. Clin. Infect. Dis. 44(1), 94-102 (2007).

5 Cattamanchi A, Smith R, Steingart KR et al. Interferon-gamma release assays for the diagnosis of latent tuberculosis infection in HIV-infected individuals: a systematic review and meta-analysis. J. Acquir. Immune Defic. Syndr. 1999. 56(3), 230-238 (2011).

6 Diel R, Loddenkemper R, Niemann S, Meywald-Walter K, Nienhaus A. Negative and positive predictive value of a wholeblood interferon- $\gamma$ release assay for developing active tuberculosis: an update. Am. J. Respir. Crit. Care Med. 183(1), 88-95 (2011).

7 Abgrall S, Del Giudice P, Melica G, Costagliola D. FHDH-ANRS CO4. $\mathrm{HIV}$-associated tuberculosis and immigration in a high-income country: incidence trends and risk factors in recent years. AIDS 24(5), 763-771 (2010).

8 Grant AD, Bansi L, Ainsworth J et al. Tuberculosis among people with HIV infection in the United Kingdom: opportunities for prevention? AIDS 23(18), 2507-2515 (2009).

9 Lodi S, del Amo J, d'Arminio Monforte A et al. Risk of tuberculosis following HIV seroconversion in high-income countries. Thorax 68(3), 207-213 (2013).

10 Karo B, Haas W, Kollan C et al. Tuberculosis among people living with HIV/AIDS in the German ClinSurv HIV Cohort: long-term incidence and risk factors. BMC Infect. Dis. 14, 148 (2014).

11 Pullar N, Steinum H, Bruun J, Dyrhol-Riise A. HIV patients with latent tuberculosis living in a low-endemic country do not develop active disease during a 2 year follow-up; a Norwegian prospective multicenter study. BMC Infect. Dis. 14(1), 667 (2014).

12 Martínez-Pino I, Sambeat MA, LacalleRemigio JR, Domingo P, VACH Cohort Study Group. Incidence of tuberculosis in $\mathrm{HIV}$-infected patients in Spain: the impact of treatment for LTBI. Int. J. Tuberc. Lung Dis. 17(12), 1545-1551 (2013).

13 Van Leth F, Kalisvaart NA, Erkens CG, Borgdoff MW. Projection of the number of patients with tuberculosis in The Netherlands in 2030. Eur. J. Public Health 19(4), 424-427 (2009). 
14 Burzynski J, Schluger NW. The epidemiology of tuberculosis in the United States. Semin. Respir. Crit. Care Med. 29(5), 492-498 (2008).

15 Mulder C, Mulleners B, Borgdorff MW, van Leth F. Predictive value of the tuberculin skin test among newly arriving immigrants. PLoS ONE 8(3), e60130 (2013).

16 Pareek M, Watson JP, Ormerod LP et al. Screening of immigrants in the UK for imported latent tuberculosis: a multicentre cohort study and cost-effectiveness analysis. Lancet Infect. Dis. 11(6), 435-444 (2011).

17 Baussano I, Nunn P, Williams B, Pivetta E, Bugiani M, Scano F. Tuberculosis among health care workers. Emerg. Infect. Dis. 17(3), 488-494 (2011).

18 Farley JE, Tudor C, Mphahlele M et al. A national infection control evaluation of drug-resistant tuberculosis hospitals in South Africa. Int. J. Tuberc. Lung Dis. 16(1), 82-89 (2012).
19 Brouwer M, Coelho E, das Dores Mosse C, van Leth F. Implementation of tuberculosis infection prevention and control in Mozambican health care facilities. Int. J. Tuberc. Lung Dis. 19(1), 44-49 (2015).

20 Brouwer M, Coelho E, Dores Mosse C das, Brondi L, Winterton L, van Leth F. Healthcare workers' challenges in the implementation of tuberculosis infection prevention and control measures in mozambique. PLoS ONE 9(12), e114364 (2014). 